8. Han J., Zhang Q., Kong F., et al. (2012). The Incidence of Invasion and Metastasis of Nasopharyngeal Carcinoma at Different Anatomic Sites in the Skull Base. The Anatomical Record, 295(8), 1252-1259.
9. Hanna E., Vural E., Prokopakis E., et al. (2007). The sensitivity and specificity of highresolution imaging in evaluating perineural spread of adenoid cystic carcinoma to the skull base. Arch Otolaryngol Head Neck Surg, 133(6), 541-545.

\title{
GIÁ TRI CHẨN ĐOÁN CỦA CộNG HƯởNG TỪ KHUẾCH TÁN VÀ CộNG HƯởNG TỪ TƯƠNG PHẢN ĐộNG HỌC TRONG PHÂN BIÊTT U TUYẾN NƯỚC BỌT MANG TAI LÀNH TÍNH VÀ ÁC TÍNH
}

\section{TÓM TẮT}

Muc tiêu: Xác định giá trị của cộng hưởng từ khuếch tán (DWI) và cộng hưởng từ tương phản động học (DCE) trong phân biệt u tuyến mang tai lành tính và ác tính. Đối tượng và phương pháp nghiên cứu: nghiên cứu được thực hiện trên 39 bệnh nhân u tuyến nước bọt mang tai với 39 tổn thương (25 lành tính, 14 ác tính) tại bệnh viện $\mathrm{K}$ trong thời gian từ tháng 6 năm 2020 đến tháng 6 năm 2021. Tiến hành đo giá trị mean $A D C$, phân tích đồ thị ngấm thuốc của từng tổn thương, từ đó xác định giá trị của DWI và DCE trong phân biệt u tuyến mang tai lành tính và ác tính. Kết quả: $U$ tuyến đa hình không có hạn chế khuếch tán trên DWI và bản đồ $A D C$. Các u ác tính, u Warthin hay u lymphoma thì có han chế khuếch tán. Giá trị ngưỡng $A D C$ giữa u tuyển đa hình và tổn thương ung thư là $1,415 \times 10^{-3} \mathrm{~mm}^{2} / \mathrm{s}$ với độ nhạy và độ đặc hiệu tương ứng là $72 \%$ và $98 \%$.Giá trị ngưỡng ADC $0.905 \times 10^{-3} \mathrm{~mm}^{2} / \mathrm{s}$ giữa khối u Warthin và tổn thương ung thư với độ nhạy và đặc hiệu tương ứng là $93 \%$ và $99 \%$. Trên DCE, khi tổn thướng có dang đồ thị loại $A$ và $D$ cho thây tổn thương là lành tính và có sự chồng lấp khi tổn thương có dạng đồ thị loại $B$ và C. Khi kết hợp DWI và DCE cho thấy khả năng phân biêt giữa tổn thương lành tính và tổn thương ác tính cải thiện đáng kể so với việc sử dụng từng phương pháp ( $\mathrm{p}<0.05)$ Kết luận: Cộng hưởing từ khuếch tán với giá trị $A D C$ kết hợp đồ thị ngấm thuốc trên cộng hưởng từ tương phản động học là một phương pháp hữu ích giúp chẩn đoán phân biệt các khối u thường gặp trong tuyến nước bọt mang tai.

Key word: u tuyến nước bọt mang tai, DWI, ADC, DCE, đô thị ngấm thuốc.

\section{SUMMARY \\ DYNAMIC CONTRAST MAGNETIC RESONANCE IMAGING (DCE-MRI) AND DIFFUSION WEIGHTED MR IMAGING (DWI) FOR}

${ }^{1}$ Trung tâm Chẩn đoán hinh ảnh- Bẹnhviện K ²Bộ môn Chẩn đoán hình ảnh - Trườngđạihọc Y HàNội Chịu trách nhiệm chính: Nguyễn Thùy Trang Email: trangnguyen2195@gmail.com Ngày nhận bài: 14.5.2021

Ngày phản biện khoa học: 7.7.2021 Ngày duyệt bài: 19.7.2021

\section{Nguyễn Thùy Trang ${ }^{1,2}$, Bùi Văn Giang ${ }^{1,2}$ \\ DIFFERENTIATION BETWEEN BENIGN AND MALIGNANT PAROTID GLAND TUMORS}

Purpose: To determine the value of adding diffusion-weighted (DW) magnetic resonance (MR) imaging to dynamic contrastmaterial-enhanced MR imaging when distinguishingbetween benign and malignant parotid tumors. Material and Methods: Theprospective study was conducted on 39 parotid salivary gland tumors with 39 lesions ( 25 benign, 14 malignant) at the National cancer hospital from June 2020 to June 2021. We evaluated themean ADC, analyze the Time-intensity curve (TIC) of each lesion, determining the value of DWI and DCE in distinguishing benign and malignant parotid tumors. Results: Pleomorphic adenomas have no diffusion restriction on DWI and ADC maps. Malignant neoplasms, Warthin tumors, or lymphomas restricted diffusion. ADC threshold values between pleomorphic adenomas and carcinomas were 1,415 $\mathrm{x} 10^{-3} \mathrm{~mm}^{2} / \mathrm{s}$ with sensitivity and specificity of $72 \%$ and $98 \%$, respectively. ADC threshold valueswere $0.905 x$ $10^{-3} \mathrm{~mm}^{2} / \mathrm{s}$ between Warthin tumors and carcinomas with sensitivity and specificity of $93 \%$ and $99 \%$, respectively. On the DCE, when the lesions have a curve of types $A$ and $D$ regarded as benign and there is an overlap when the lesions have a curve of type $B$ and $C$. The combination of DWI and DCE improves the differentiation between benign and malignant lesions significantly compared with using each method ( $p<$ 0.05). Conclusion: Diffuse magnetic resonance with $A D C$ value combined with dynamic contrast magnetic resonance imaging is a useful method for differential diagnosis of common tumors in the parotid glands.

Keywords: parotid gland tumors, DWI, ADC, DCE, Time-intensity curve (TIC)

\section{I. ĐẶT VẤN ĐỀ}

Chẩn đoán trước mổ u tuyến nước bot mang tai đóng vai trò quan trọng trong việc lập kế hoạch phẫu thuật vì các phẩu thuật viển cần sử dụng một cách tiếp cận trực quan để điều trị các khối u ác tính. Ngoài ra, việc chẩn đoánđúng trước phẫu thuật giúp giảm thiểu các can thiệp không cần thiết ở những bệnh nhân có khối u lành tính, đặc biệt là những bệnh nhân có nhiều yếu tố nguy cơ. Chọc hút tế bào bằng kim nhỏ là 
một thủ thuật xâm lấn tối thiểu được sử dụng tương đối phổ biến trongchẩn đoán u tuyến nước bọt mang tai trước phẫu thuật; tuy nhiên, kết quả tế bào học chọc hút bằng kim nhỏ không phải lúc nào cũng đưa ra được chẩn đoán chính xác về bản chất của khối u bởi vì đôi khi bệnh phẩm kim nhỏ thu được không đủ chẩn đoánbởi kích thước mẫu quá nhỏ hoặc do vị trí sâu của khối u ${ }^{1}$.

Các nghiên cứu chỉ ra rằng gần như $25 \%$ u tuyến đa hình không được điều trị có biểu hiện thoái hóa ác tính, thường là ung thư biểu mô tuyến thoái triển từ u tuyến đa hình². Mặt khác, sự phát triển của bệnh ác tính ở bệnh nhân có khối u Warthin là tương đối hiếm xảy ra với ít hơn 1\% bệnh nhân². Ngoài ra, phẫu thuật cắt bỏ toàn bộ khối u, với đủ các thành phần của khối bao gồm cả phần vỏ của khối u, đã được khuyến cáo đối với bệnh nhân u tuyến đa hình để giảm tỷ lệ tái phát tại chỗ ${ }^{3}$. Do đó, kế hoạch điều trị khác nhau tùy thuộc vào bản chất của khối u.

Các đặc điểm hình ảnh MR thường quy của khối u tuyến nước bọt ác tính được báo cáo là bờ khối u không đều, tín hiệu không đồng nhất và sự xâm lấn nhu mô xung quanh ${ }^{2}$. Trong nghiên cứu của Som và Biller ${ }^{4}$ nhận thây rằng các khối u ác tính của tuyến nước bọt mang tai thường có đường bờ không xác định và cường độ tín hiệu thấp trên cả $T 1$ và $T 2$. Ngược lại, Freling và cộng sự $5^{5}$ đã báo cáo rằng đường bờ của khối $u$, tính đồng nhất và cường độ tín hiệu không phải là yếu tố phân biệt để dự đoán chính xác bệnh lành tính hoặc ác tính.

Hình ảnh cộng hưởng từ tương phản động học cho thây giá trị cao trong việc xác định đặc điểm của các khối u tuyến nước bọt ${ }^{6}$. Ngoài ra, cộng hưởng từ khuếch tán (DWI)đã được báo cáo là rất có giá trị?. Nghiên cứu của Eida và cộng sựicho thấy rằng bản đồ khuếch tán biểu kiến ( $A D C$ map) biểu thị các khu vực có $A D C$ cao $\left(1,8 \times 10^{\wedge} 3 \mathrm{~mm} 2 /\right.$ giây $)$ có tỷ lệ u lành tính nhiều hơn đáng kể so với u ác tính ở tuyến nước bọt mang tai. Tuy nhiên, giá trị chẩn đoán của sự kết hợp giữa hình ảnh cộng hưởng từ khuếch tán và hình ảnh cộng hưởng từ tương phản động học trong mô tả đặc điểm của khối u mang tai đã không đã được nhấn mạnh trước đây trong các tài liệu. Mục đích nghiên cứu của chúng tôi là xác định giá trị của việc kết hợp cộng hưởng từ khuếch và cộng hưởng từ tương phản động học trongchẩn đoán phân biệt giữa u tuyến nước bọt mang tai lành tính và ác tính.

\section{II. ĐỐI TƯỢNG VÀ PHƯƠNG PHÁP NGHIÊN CỨU}

2.1 Đối tượng nghiên cứu: Nghiên cứu trên 39 bệnh nhẩn được chẩn đoán u tuyến nước bơt mang tai và được chụp cộng hưởng từ, được phẫu thuật và được làm mô bệnh học tại bệnh viện $\mathrm{K}$ cơ sở Tân Triều từ tháng 6 năm 2020 đến tháng 6 năm 2021.

2.2 Phương pháp nghiên cứu: Mô tả cắt ngang tiến cứu.

Các bệnh nhân đều được chụp $\mathrm{CHT}$, trong đó 39 bệnh nhân có sử dụng chuối xung DWI,12 bệnh nhân có sử dụng đồng thời cả chuỗi xung DWI và $D C E$. Các giá trị $A D C$ và đồ thị được đo trên trạm xử lý hình ảnh Ziostation2-Version 2.9.7.2 của Ziosoft, Tokyo office. Trên bản đồ ADC, Tiến hành đặt 3 ROI tại phần mô đặc của u, tránh các vùng hoại tử, hóa nang, xuất huyết, ngấm vôi, mạch máu từ đó tìm ra mean $A D C$

Để thu được đồ thị ngấm thuốc thì ROI được đặt ở phần u đặc, không thay đổi vị trí qua các lần chụp. Các dạng đường cong ngấm thuốc theo thời gian bao gồm: loại $A$ : thời gian đạt đỉnh cao hơn 120 giây; loại B: thời gian đạt đỉnh là 120 giây hoặc ít hơn với tỷ lệ thải thuốc cao $(>30 \%)$; loại C: thời gian đạt đỉnh là 120 giây hoặc ít hơn với tỷ lệ thải thuốc thấp $(<30 \%)$; và loại $D$ : đồ thị đingang (không ngấm thuốc).

2.2 Phương tiện nghiên cứu: Máy cộng hưởng từ 1.5 Tesla tại bệnh viện $\mathrm{K}$ cơ sở Tân Triều.

2.4 Phân tích số liệu: Sử dụng phương pháp thống kê toán học trong u học với phần mềm SPSS 22.0

Mô tả các biến liên tục và so sánh giữa hai nhóm bằng kiểm định độc lập $\mathrm{t}$-test. Các biến phân loại được so sánh bằng kiểm định chi bình phương

Sử dụng đường cong ROC để so sánh giá trị chẩn đoán của biến, đồng thời tính được ngưỡng tối ưu theo chỉ số Youden

\section{KẾT QUẢ NGHIÊN CỨU}

Bảng 1: Dạng đồ thị của các loại u hay gặp tại tuyến nước bọt mang tai

\begin{tabular}{|c|c|c|c|c|c|c|}
\hline Loại đồ thị & U tuyến đa hình & U Warthin & Ung thư & nang & Tổng & p \\
\hline Loại A & 3 & 0 & $\mathbf{0}$ & $\mathbf{0}$ & $\mathbf{3}$ & \\
\hline Loại B & 0 & 3 & $\mathbf{1}$ & $\mathbf{0}$ & $\mathbf{3}$ & 0,000 \\
\hline Loại C & 1 & 0 & $\mathbf{4}$ & $\mathbf{0}$ & $\mathbf{5}$ \\
\hline Loại D & 0 & 0 & $\mathbf{0}$ & $\mathbf{1}$ & $\mathbf{1}$ \\
\hline
\end{tabular}


Bảng 2: Giá trị ADC của các loại u thường gặp tại tuyến nước bọt mang tai trên bản đồ ADC

\begin{tabular}{|c|c|c|c|c|c|}
\hline Mean ADC & $\begin{array}{c}\text { U tuyến đa } \\
\text { hình }\end{array}$ & U Warthin & Ung thư & lymphoma & Tổng \\
\hline $\bar{A} \pm S D$ & $1,93 \pm 0,32$ & $0,83 \pm 0,06$ & $0,95 \pm 0,11$ & $0,53 \pm 0,11$ & $2,3 \pm 0,28$ \\
\hline
\end{tabular}

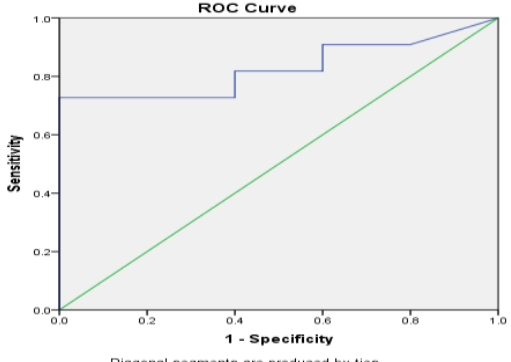

Hình 1. Đường cong ROC thể hiện giá trị cutoff của ADC giữa ung thư biểu mô và u Warthin tại tuyến nước bọt mang tai.

Diện tích dưới đường cong ROC 0,827

Chọn cutoff là 0,905 với $\mathrm{J}=0,727$ (ung thư giá trị 1 , warthin giá trị O)

Đô nhạy $72 \%$

Độ đặc hiệu $98 \%$.

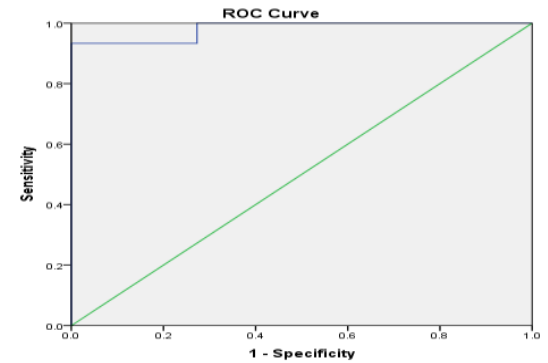

Hình 2. Đường cong ROC thể hiện giá trị cutoff của $A D C$ giữa ung thư biểu mô và u tuyến đa hinh tại tuyến nước bọt mang tai.

Diện tích dưới đường cong 0,982

Chọn cutoff $=1,415$ với $\mathrm{J}=0,933$ (u đa hình

là 1 , ung thư là 0 )

Độ nhay $93 \%$

Độ đặc hiệu 99\%

\begin{tabular}{|c|c|c|c|c|c|}
\hline Đồ thị loại A & \multicolumn{2}{|c|}{ Đồ thị loại B } & \multicolumn{2}{c|}{ Đồ thị loại C } & Đồ thị loại D \\
\hline \multirow{4}{*}{} & \multicolumn{2}{|c|}{ ADC } & \multicolumn{2}{|c|}{ ADC } & \\
\cline { 2 - 6 } & $>=1.415$ & $<1,415$ & $>=0.905$ & $<0.905$ \\
\cline { 2 - 6 } & & & & & \\
\hline
\end{tabular}

Hinh 3. Lưu đồ chấn đoán các loại u thường gặp tại tuyến nước bọt mang tai theo đồ thị ngấm thuốc và giá trị $A D C$

Khi chỉ chẩn đoán phân biệt khối u lành tính và ác tính dựa trên cơ sở của các loại đồ thị đơn thuần, các khối u có đồ thị loại $A, B$ và $D$ được coi là khối u lành tính và khối u có đồ thị loại $C$ là khối u ác tính, độ nhạy, độ đặc hiệu và giá trị dự đoán dương tính và âm tính là $80 \%$ (4 trên 5 tổn thương), 87,5\% (7 trên 8 tổn thương), 87\% (7 trong số 8 tổn thương), $80 \%$ (4 trong số 5 tổn thương). Khi đánh giá các khối u có kết hợp giá trị $A D C$ với các đồ thị loại $B$ và $C$ và sửa đổi chẩn đoán với tham chiếu đến các giá trị $A D C$, thì các u đều được chẩn đoán đúng về mặt bản chất mô học, cải thiện chẩn đoán rõ rệt (khác biệt có nghĩa thống kê với $\mathrm{p}$ <0.05). Khi chúng tôi kết hợp các giá trị $A D C$ với tất cả các loại đồ thị và sửa đổi chẩn đoán với tham chiếu đến các giá trị $A D C$, thì các tổn thương cũng đều mang lại chẩn đoán đúng về mô học nhưng sự khác biệt không có ý nghĩa thống kê ( $p>0.05)$.

\section{BÀN LUÂ̂N}

Trong nghiên cứu của chúng tôi đã chỉ ra giá trị meanADC của các loại u tuyến mang tai thường gặp. Giá trị trung bình meanADC của tổn thương ung thư là $0,95 \pm 0,11 \times 10^{-3} \mathrm{~mm}^{2} / \mathrm{s}$. Giá tri trung bình meanADC của tổn thương $u$ tuyến đa hình là $1,93 \pm 0,32 \times 10^{-3} \mathrm{~mm}^{2} / \mathrm{s}$. Giá trị trung bình meanADC của tổn thương $u$ Warthin là $0,83 \pm$ $0,06 \times 10^{-3} \mathrm{~mm}^{2} / \mathrm{s}$. Giá trị trung bình meanADC của tổn thương u lymphoma là $0,53 \pm 0,11 \times 10$ ${ }^{3} \mathrm{~mm}^{2} / \mathrm{s}$. Các giá trị này tương đồng với các nghiên cứu của Eida và cồng sựđđã đánh giá bản đồ ADC cho 31 khối u tuyến mang tai (22 khối u lành tính và 9 khối u ác tính). Cũng như các nghiên cứu khác như nghiên cứu của Haberman và cộng sự ${ }^{10}$ đều đem lại kết quả khá tương đồng.

Nghiên cứu chúng tôi cho thấy rằng việc sử dụng cộng hưởng từ tương phản động học có thể cải thiện độ chính xác chẩn đoán trong việc xác đinh đắc điểm lành tình ác tính của các khối u tuyến nước bot mang tai. Trong nghiên cứu củachúng tôi cho kết quả rằng hầu hết các u tuyến đa hình đều có dạng đồ thị ngấm thuốc loại $A$. Đồ thị ngấm thuốc loại $C$ thường là $u$ Warthin và một số ung thư biểu mô. Đồ thị 
ngấm thuốc loại $B$ chủ yếu là các tổn thương ung thư biểu mô, ngoài ra có một vài u tuyến đa hình. Đồ thị ngấm thuốc loại D là đồ thị đi ngang biểu thị sự không ngấm thuốc của tổn thương nang. Do đó, ở những bệnh nhân có đồ thị ngấm thuốc loại $C$, chúng ta phải phân biệtung thư biểu mô với khối u Warthin; và, ở những bệnh nhân có khối u có đồ thị ngấm thuốc loại $B$, chúng ta phải phân biệtung thư biểu mô với u tuyến đa hình.Việc kết hợp giá trị ADC đặc biệt hữu ích khi phân biệt giữa các khối u tuyến nước bọt mang tai có biểu đồ ngấm thuốc loại $B$ và $C$.

Từ đây, chúng tôi sử dụng đường cong ROC để so sánh giá trị chẩn đoán, đồng thời tính được ngưỡng tối ưu theo chỉ số Youden để tìm giá trị $A D C$ ngưỡng giữa ung thư biểu mô với khối u Warthin là $0,905 \times 10^{-3} \mathrm{~mm}^{2} / \mathrm{s}$ và giá trị ADC để phân biệt giữa u tuyến đa hình với ung thư biểu mô là $1,415 \times 10^{-3} \mathrm{~mm}^{2} / \mathrm{s}$.U Warthin thường có giá trị $A D C$ thấp hơn ung thư biểu mô, còn u tuyến đa hình lại có giá trị $A D C$ cao hơn ung thư biểu mô và sự khác biệt có ý nghĩa thống kê. Giá trị ADC ngường để phân biệt giữa u tuyến đa hình và ung thư biểu mô là tương đồng với các nghiên cứu khác trên thế giới như Yabuchi và cộng sự ${ }^{6}$, Eida và cộng sự ${ }^{9}$. Tuy nhiên giá trị ADC ngưỡng để phân biệt giữa u Warthin và ung thư biểu mô lại thấp hơn so với các giá trị của Yabuuchi ${ }^{6}\left(1,01 \times 10^{-3} \mathrm{~mm}^{2} / \mathrm{s}\right)$ nhưng sự khác biệt này là không có ý nghĩa thống kê và sự khác biệt có thể do phương pháp của từng nghiên cứu là khác nhau và hiện cũng chưa có sự thống nhất giữa các nghiên cứu.

Với sự trợ giúp củagiá trị $A D C$ ngưỡng, chúng tôi đã sửa đổi chính xác chẩn đoán của một u tuyến đa hình có đồ thị ngấm thuốc loại $B$ và một ung thư biểu mô có đồ thị ngấm thuốc loại C. Để giải thích một cách phù hợp cho kết quả này là mặc dù các u tuyến đa hình thường cóđồ thị ngấm thuốc loại $A$ và có mật độ tế bào tương đối cao. Tuy nhiên mật độ tế bào của u tuyến đa hình vẫn nhỏ hơn hởn so với ung thư biểu mô. Cả ung thư biểu mô và khối u Warthin có mật độ tế bào rất $\mathrm{cao}^{2}$; nhưng mật độ tế bào của ung thư biểu mô nhỏ hơn khối u Warthin. Trong nghiên cứu của chúng tôi, chẩn đoán giá trị không cải thiện đáng kể khi chúng tôi áp dụng các ngưỡng giá trị $A D C$ trong tất cả các khối u với 4 loại đồ thị ngấm thuốc.

Nghiên cứu của chúng tôi đã có một số hạn chế. Đầu tiên, cõ̃ mẫu của chúng tôi chưa đủ lớn, số lượng tổn thương được chụp cộng hưởng từ tương phản động học còn ít, nên tính chất đại diện chưa cao. Vì thế cần có thêm nhiều nghiên cứu chuyên sâu khác với cỡ mẫu lớn hơn để so sánh cũng như đưa ra được khuyến cáo cần thiết đối với việc đánh giá tính chất lành tính và ác tính trên hình ảnh cộng hưởng từ. Thứ hai, khả năng tái tạo giá trị $A D C$ giữa các giá trị $b$ vẫn còn tranh cãi. Như vậy, giá trị $A D C$ ngưỡng có được từ kết quả của chúng tồi cần được cân nhắc trước khiáp dụngcho các nghiên cứu khác.

\section{KẾT LUÂ̂N}

Tóm lại, cộng hưởng từ khuếch tán với giá trị ADC kết hợp đồ thị ngấm thuốc trên cộng hưởng từ tương phản động học là một phương pháp hữu ích giúp chẩn đoán phân biệt các khối u thường gặp trong tuyến nước bọt mang tai.

\section{TÀI LIÊU THAM KHẢO}

1. Zbären P, Nuyens $M$, Loosli $H$, Stauffer $E$. Diagnostic accuracy of fine-needle aspiration cytology and frozen section in primary parotid carcinoma: Diagnoses of Parotid Carcinomas. Cancer. doi:10.1002/cncr.20186

2. Som PM, Biller HF. High-Grade Malignancies of the Parotid Gland: Identification with MR Imaging. Radiology 1989;173:823-826.

3. Witt RL. The Significance of the Margin in Parotid Surgery for Pleomorphic Adenoma: The Laryngoscope. $\quad 2002 ; 112(12): 2141-2154$. doi:10.1097/00005537-200212000-00004

4. Som PM, Biller HF. High-grade malignancies of the parotid gland: identification with MR imaging. Radiology. doi:10.1148/radiology.173.3.2813793

5. Freling NJ, Molenaar WM, Vermey A, et al. Malignant parotid tumors: clinical use of MR imaging and histologic correlation. Radiology. 1992;185(3):691-696. doi:10.1148/radiology.185.3.1438746

6. Yabuuchi $H$, Matsuo $Y$, Kamitani $T$, et al. Parotid Gland Tumors: Can Addition of Diffusionweighted MR Imaging to Dynamic Contrastenhanced MR Imaging Improve Diagnostic Accuracy in Characterization? Radiology. 2008; 249(3):909-916. doi:10.1148/radiol.2493072045

7. Motoori $K$, Iida $Y$, Nagai $Y$, et al. MR Imaging of Salivary Duct Carcinoma. Published online 2005:6.

8. Eida S, Sumi M, Sakihama $\mathbf{N}$, Takahashi $H_{\text {, }}$ Nakamura T. Apparent Diffusion Coefficient Mapping of Salivary Gland Tumors: Prediction of the Benignancy and Malignancy. Am J Neuroradiol. 2007;28(1):116-121.

9. Eida S, Sumi M, Nakamura T. Multiparametric magnetic resonance imaging for the differentiation between benign and malignant salivary gland tumors. J Magn Reson Imaging. 2010;31(3):673679. doi:10.1002/jmri.22091

10. Habermann CR, Gossrau P, Graessner J et al. Diffusion-Weighted Echo-Planar MRI: A Valuable Tool for Differentiating Primary Parotid Gland Tumors? RöFo - Fortschritte Auf Dem Geb Röntgenstrahlen Bildgeb Verfahr. 2005;177(07):940945. doi:10.1055/s-2005-858297 